\title{
Asymptotic representation of low- and intermediate-degree $p$-modes in stars
}

\author{
P. Smeyers $\star$ \\ Instituut voor Sterrenkunde, Katholieke Universiteit Leuven, Celestijnenlaan 200 B, 3001 Heverlee, Belgium
}

Received 27 February 2003 / Accepted 16 May 2003

\begin{abstract}
A first-order asymptotic representation is developed for low- and intermediate-degree $p$-modes in stars for which the lower boundary of the resonant acoustic cavity is not located close to the star's centre. To this end, a fourth-order system of differential equations in the radial parts of the divergence and the radial component of the Lagrangian displacement is adopted. The lower boundary of the resonant acoustic cavity is considered to be a turning point for one of the differential equations. As in a previous asymptotic study of low-degree $p$-modes with high radial orders, asymptotic expansion procedures applying to self-adjoint second-order differential equations with a large parameter are used by extension of these methods. The main result is that, in contrast with the usual first-order asymptotic theory for low-degree $p$-modes of high radial orders, the present firstorder asymptotic representation leads to small frequency separations $D_{n, \ell}$ different from zero. The validity of the asymptotic representation is tested for $p$-modes of the equilibrium sphere with uniform mass density, since the modes of this model are determined by means of exact analytical solutions.
\end{abstract}

Key words. stars: oscillations - methods: analytical

\section{Introduction}

In stars, $p$-modes are thought to originate from interferences of acoustic waves which propagate to-and-fro in a resonant cavity inside the star. The resonant cavity is bounded above by the rapid decrease of the sound velocity near the star's surface and below by the increase of the sound velocity with depth. The radial distance of the lower boundary from the star's centre decreases with the frequency of the $p$-mode and increases with the degree of the spherical harmonic to which the mode belongs.

For low-degree and higher-order $p$-modes, Tassoul developed a second-order asymptotic theory, first with the use of the Cowling approximation (Tassoul 1980), and afterwards without the use of this approximation (Tassoul 1990).

Smeyers et al. (1996) reconsidered the second-order asymptotic theory by using asymptotic expansion procedures which apply to singular perturbation problems (Kevorkian \& Cole 1981, 1996) and derived an asymptotic representation similar to that developed by Tassoul, apart from a small difference which does not affect the results. In their procedure, Smeyers et al. divided the star into three regions: the region away from the boundary points at $r=0$ and $r=R$, and the two boundary layers near the boundary points. In the first region, asymptotic solutions for the functions considered are constructed that are oscillatory, while in the boundary layers non-oscillatory asymptotic solutions are constructed. From a

\footnotetext{
^ e-mail: Paul.Smeyers@ster.kuleuven.ac.be
}

physical point of view, the region away from the boundary points can be assimilated with the resonant acoustic cavity, where acoustic waves are propagating, and the two boundary layers can be assimilated with the regions outside the acoustic cavity, where the acoustic waves are attenuated or evanescent. In this procedure, the lower boundary of the acoustic cavity is tacitly assumed to be located close to the star's centre (for a similar remark, see Lopes \& Turck-Chièze 1994, Sect. 2.2.2). The assumption is justified for low-degree $p$-modes of very large radial orders but is highly questionable for low-degree $p$-modes of less large radial orders and for intermediate-degree $p$-modes.

In this paper, our aim is to develop a first-order asymptotic representation of low- and intermediate-degree $p$-modes for which the lower boundary of the resonant acoustic cavity is not located close to the star's centre. We again start from the fourth-order system of differential equations in the radial parts of the divergence and the radial component of the Lagrangian displacement, which stems from Pekeris (1938) and was readopted by Tassoul (1990). We consider the lower boundary of the resonant acoustic cavity to be a turning point for one of the differential equations.

In the resonant acoustic cavity, we distinguish between the region away from the boundaries of the cavity and the two small regions near these boundaries. Outside the resonant acoustic cavity, we distinguish between the small region near the transition point and the region away from that point, which extends to the star's centre. In each region, we apply an 
adequate asymptotic expansion procedure for the construction of the asymptotic solution.

This first-order asymptotic representation yields asymptotic approximations for the small frequency separations $D_{n \ell}$ that are different from zero, in contrast with what the first-order asymptotic representation for low-degree $p$-modes of high radial orders does. We illustrate our asymptotic representation of $p$-modes with results for the equilibrium sphere with uniform mass density as was already done by Tassoul (1990), since this is the only equilibrium model for which eigenfrequencies and small frequency separations can be determined with great accuracy even for higher-degree modes.

The plan of the paper is as follows. In Sect. 2, we present the basic equations. In Sect. 3, we construct the asymptotic solution in the region in the resonant acoustic cavity that is away from the boundaries. The asymptotic solutions in the small regions near the lower boundary of the resonant acoustic cavity are constructed in Sects. 4 and 5. In Sect. 6, we construct the asymptotic solution in the region outside the acoustic cavity that is away from the lower boundary of the cavity and extends to the star's centre. In Sect. 7, the asymptotic solution valid in the boundary layer near $r=R$ is constructed and the eigenfrequency equation is derived. In Sect. 8, we apply the asymptotic representation to low- and intermediate-degree $p$-modes in the equilibrium sphere with uniform mass density.

\section{Basic equations}

Consider a spherically symmetric and static star with mass $M$ and radius $R$ that is subject to an oscillation mode $p$ of a low or an intermediate degree $\ell$. As usual, we introduce a system of spherical coordinates $r, \theta, \phi$ defined in an inertial frame of reference whose origin coincides with the star's mass centre. Using conventional notations, we write the fourth-order system of governing differential equations in the radial parts of the divergence and the radial component of the Lagrangian displacement, respectively $\alpha(r)$ and $\xi(r)$, in the form

$$
\begin{aligned}
& \frac{\mathrm{d}^{2} \alpha}{\mathrm{d} r^{2}}+K_{2}(r) \frac{\mathrm{d} \alpha}{\mathrm{d} r}+\left[\frac{1}{\varepsilon^{2}} \varphi(r)+K_{3}^{*}(r)+\varepsilon^{2} K_{1}(r)\right] \alpha \\
& =-K_{4}(r) \frac{\mathrm{d} \xi}{\mathrm{d} r} \\
& \frac{\mathrm{d}^{2} \xi}{\mathrm{d} r^{2}}+\frac{4}{r} \frac{\mathrm{d} \xi}{\mathrm{d} r}-\frac{\ell(\ell+1)-2}{r^{2}} \xi \\
& =\frac{\mathrm{d} \xi}{\mathrm{d} r}+\left[\frac{2}{r}-\varepsilon^{2} \frac{c^{2}(r)}{g(r)} K_{1}(r)\right] \alpha
\end{aligned}
$$

where

$$
\begin{aligned}
& \varepsilon=\frac{1}{|\sigma|}, \\
& \varphi(r)=\frac{1}{c^{2}(r)}-\frac{(\ell+1 / 2)^{2}}{\sigma^{2} r^{2}}, \\
& K_{1}(r)=\ell(\ell+1) \frac{N^{2}}{r^{2}}, \\
& K_{2}(r)=\frac{2}{r}+\frac{2}{\rho c^{2}} \frac{\mathrm{d}\left(\rho c^{2}\right)}{\mathrm{d} r}-\frac{1}{\rho} \frac{\mathrm{d} \rho}{\mathrm{d} r},
\end{aligned}
$$

$$
\begin{aligned}
K_{3}^{*}(r)= & \frac{1}{4} \frac{1}{r^{2}}+\frac{2 g}{c^{2}}\left(\frac{1}{g} \frac{\mathrm{d} g}{\mathrm{~d} r}+\frac{1}{r}\right) \\
& +\frac{1}{\rho c^{2}} \frac{\mathrm{d}\left(\rho c^{2}\right)}{\mathrm{d} r}\left(\frac{2}{r}-\frac{1}{\rho} \frac{\mathrm{d} \rho}{\mathrm{d} r}\right)+\frac{1}{\rho c^{2}} \frac{\mathrm{d}^{2}\left(\rho c^{2}\right)}{\mathrm{d} r^{2}} \\
K_{4}(r)= & -\frac{2 g}{c^{2}}\left(\frac{1}{g} \frac{\mathrm{d} g}{\mathrm{~d} r}-\frac{1}{r}\right)
\end{aligned}
$$

In these equations, $1 / \varepsilon^{2}$ is considered to be a large parameter. Equation (1) differs from Eq. (2) in Smeyers et al. (1996) by the replacement of the coefficient of the large parameter, $c^{-2}(r)$, by the coefficient $\varphi(r)$, and by the associated change of the function $K_{3}(r)$ into the function $K_{3}^{*}(r)$. The equation has a turning point or a transition point at the zero of the coefficient $\varphi(r)$ of the large parameter, whose radial coordinate $r_{\mathrm{t}}$ is determined by the equation

$r_{\mathrm{t}}=\frac{(\ell+1 / 2) c\left(r_{\mathrm{t}}\right)}{\sigma}$

We consider the turning point to be located outside the boundary layer near $r=0$.

The adoption of the parameter $(\ell+1 / 2)^{2}$ in Definition (4) of the function $\varphi(r)$, and not that of the parameter $\ell(\ell+1)$, is dictated by the requirement of constructing first-order asymptotic solutions for the function $\alpha(r)$ that have the right behavior as $r \rightarrow 0$. That $(\ell+1 / 2)^{2}$ is the correct choice of the parameter was emphasized earlier by Roxburgh \& Vorontsov (1996) relative to the finiteness of the second-order terms in asymptotic expansions in terms of Airy functions that apply to solar acoustic oscillations of low and intermediate degree (see the comments of these authors below their Eq. (15)). The authors added the comment:

It is well known that in quantum mechanics the replacement of $\ell(\ell+1)$ by $(\ell+1 / 2)^{2}$ improves the accuracy of JWKB formulae for the potentials of the type $\ell(\ell+1) / r^{2}$ (Kemble 1937; Langer 1937).

In their second-order treatment, Roxburgh \& Vorontsov adopted an approach used by Vorontsov (1991), which consists in developing a composite description rather than a purely asymptotic one: they derived the eigenfrequency equation by matching asymptotic solutions that are valid in the solar interior, with "exact" non-asymptotic solutions that are valid near the surface. The solutions in the deep interior were constructed as asymptotic solutions of a single second-order differential equation for a variable related to the Eulerian perturbation of the pressure. In this equation, the dominant effects of the Eulerian perturbation of the gravitational potential and its first derivative were taken into consideration.

\section{The asymptotic solution in the region in the resonant acoustic cavity away from its boundaries}

In the region in the acoustic cavity away from the turning point at $r=r_{\mathrm{t}}$ and from the star's boundary at $r=R$, we proceed as in Smeyers et al. (1996): we use, by extension, 
a two-variable expansion procedure described by Kevorkian \& Cole $(1981,1996)$ that applies to self-adjoint second-order differential equations with a large parameter. We adopt the fast variable

$\tau(r)=\frac{1}{\varepsilon} \int_{r_{\mathrm{t}}}^{r} \sqrt{\varphi\left(r^{\prime}\right)} \mathrm{d} r^{\prime}$

and consider the radial coordinate $r$ as the slow variable. By introducing asymptotic expansions for the functions $\alpha(r)$ and $\xi(r)$ of the form

$$
\left.\begin{array}{rl}
\alpha^{(\mathrm{o})}(r ; \varepsilon)= & \alpha_{0}^{(\mathrm{o})}(\tau, r)+\varepsilon \alpha_{1}^{(\mathrm{o})}(\tau, r)+\varepsilon^{2} \alpha_{2}^{(\mathrm{o})}(\tau, r) \\
& +O\left(\varepsilon^{3}\right) \\
\xi^{(\mathrm{o})}(r ; \varepsilon)= & \xi_{0}^{(\mathrm{o})}(\tau, r)+\varepsilon \xi_{1}^{(\mathrm{o})}(\tau, r)+\varepsilon^{2} \xi_{2}^{(\mathrm{o})}(\tau, r) \\
& +O\left(\varepsilon^{3}\right),
\end{array}\right\}
$$

and, mutatis mutandis, solving the equations of the lowest orders in $\varepsilon$ as in Smeyers et al. (1996), one derives the first-order asymptotic solutions

$$
\begin{aligned}
& \alpha^{(\mathrm{o})}(r ; \varepsilon)=h(r)\left(A_{0}^{*} \cos \tau+B_{0}^{*} \sin \tau\right), \\
& \xi^{(\mathrm{o})}(r ; \varepsilon)=C_{0}^{*} r^{\ell-1}+D_{0}^{*} r^{-(\ell+2)},
\end{aligned}
$$

where

$h(r)=\left[\varphi^{1 / 4}(r) r \sqrt{\rho(r)} c^{2}(r)\right]^{-1}$,

and $A_{0}^{*}, B_{0}^{*}, C_{0}^{*}, D_{0}^{*}$ are undetermined constants. The first-order asymptotic solution for $\alpha^{(\mathrm{o})}(r ; \varepsilon)$ is oscillatory in the fast variable $\tau$, while the first-order asymptotic solution for $\xi^{(o)}(r ; \varepsilon)$ depends only on the slow variable $r$ and is non-oscillatory. The oscillatory solution for $\xi(r)$ does not appear before the secondorder asymptotic approximation.

\section{The asymptotic solution in the region near the turning point located on the inside of the acoustic cavity}

In the small region near the turning point located on the inside of the acoustic cavity, we again follow a procedure described by Kevorkian \& Cole $(1981,1996)$. In order to consider the behavior of Eq. (1) near the turning point, we introduce the coordinate

$s=r-r_{\mathrm{t}}$,

and the Taylor expansions about $s=0$ of the form

$\left.\begin{array}{ll}c(r)=c_{\mathrm{t}}+c_{1} s+O\left(s^{2}\right), & \varphi(r)=\varphi_{\mathrm{t}} s+O\left(s^{2}\right), \\ K_{1}(r)=K_{1, \mathrm{t}}+O(s), & K_{2}(r)=K_{2, \mathrm{t}}+O(s), \\ K_{3}^{*}(r)=K_{3, \mathrm{t}}^{*}+O(s), & K_{4}(r)=K_{4, \mathrm{t}}+O(s),\end{array}\right\}$

where the coefficient $\varphi_{\mathrm{t}}$ is determined as

$\varphi_{\mathrm{t}}=\frac{2}{c_{\mathrm{t}}^{2}}\left(\frac{1}{r_{\mathrm{t}}}-\frac{c_{1}}{c_{\mathrm{t}}}\right)$ and is positive. By the introduction of the coordinate

$s^{*}=\frac{s}{\delta(\varepsilon)}$,

where the function $\delta(\varepsilon) \rightarrow 0$ as $\varepsilon \rightarrow 0$, and expansions of the form

$$
\left.\begin{array}{rl}
\alpha^{(\mathrm{t})}(r ; \varepsilon)= & \mu_{0}^{(\mathrm{t})}(\varepsilon) \alpha_{0}^{(\mathrm{t})}\left(s^{*}\right)+\mu_{1}^{(\mathrm{t})}(\varepsilon) \alpha_{1}^{(\mathrm{t})}\left(s^{*}\right) \\
& +\ldots, \\
\xi^{(\mathrm{t})}(r ; \varepsilon)= & v_{0}^{(\mathrm{t})}(\varepsilon) \xi_{0}^{(\mathrm{t})}\left(s^{*}\right)+v_{1}^{(\mathrm{t})}(\varepsilon) \xi_{1}^{(\mathrm{t})}\left(s^{*}\right) \\
& +\ldots,
\end{array}\right\}
$$

Eq. (1) takes the form

$$
\begin{aligned}
\mu_{0}^{(\mathrm{t})}(\varepsilon)\left[\frac{1}{\delta^{2}(\varepsilon)} \frac{\mathrm{d}^{2} \alpha_{0}^{(\mathrm{t})}}{\mathrm{d} s^{* 2}}+\frac{1}{\delta(\varepsilon)} K_{2, \mathrm{t}} \frac{\mathrm{d} \alpha_{0}^{(\mathrm{t})}}{\mathrm{d} s^{*}}\right. \\
\left.+\frac{\delta(\varepsilon)}{\varepsilon^{2}} \varphi_{\mathrm{t}} s^{*} \alpha_{0}^{(\mathrm{t})}+\ldots\right]+\mu_{1}^{(\mathrm{t})}(\varepsilon)[\ldots]+\ldots \\
+\frac{v_{0}^{(\mathrm{t})}(\varepsilon)}{\delta(\varepsilon)} K_{4, \mathrm{t}} \frac{\mathrm{d} \xi_{0}^{(\mathrm{t})}}{\mathrm{d} s^{*}}+\ldots=0 .
\end{aligned}
$$

Within the terms containing the function $\mu_{0}^{(\mathrm{t})}(\varepsilon)$, the term involving the second derivative $\mathrm{d}^{2} \alpha_{0}^{(\mathrm{t})} / \mathrm{d} s^{* 2}$ is of the same order in $\varepsilon$ as the term involving the product $\varphi_{\mathrm{t}} s^{*} \alpha_{0}^{(\mathrm{t})}$, when

$\delta(\varepsilon)=\varepsilon^{2 / 3}$.

If the function $\mu_{0}^{(\mathrm{t})}(\varepsilon)$ is of a smaller order in $\varepsilon$ than the product $\varepsilon^{2 / 3} v_{0}^{(\mathrm{t})}(\varepsilon)$, the dominant equation in the small region near the turning-point is

$\frac{\mathrm{d}^{2} \alpha_{0}^{(\mathrm{t})}}{\mathrm{d} s^{* 2}}+\varphi_{\mathrm{t}} s^{*} \alpha_{0}^{(\mathrm{t})}=0$.

Its general solution can be expressed in terms of Airy functions as

$\alpha_{0}^{(\mathrm{t})}\left(s^{*}\right)=A_{0, \mathrm{t}} \operatorname{Ai}\left(-\varphi_{\mathrm{t}}^{1 / 3} s^{*}\right)+B_{0, \mathrm{t}} \operatorname{Bi}\left(-\varphi_{\mathrm{t}}^{1 / 3} s^{*}\right)$,

where $A_{0, \mathrm{t}}$ and $B_{0, \mathrm{t}}$ are undetermined constants. Hence, the firstorder asymptotic solution for $\alpha^{(\mathrm{t})}(r ; \varepsilon)$ takes the form

$\alpha^{(\mathrm{t})}(r ; \varepsilon)=\mu_{0}^{(\mathrm{t})}(\varepsilon)\left[A_{0, \mathrm{t}} \operatorname{Ai}\left(-\varphi_{\mathrm{t}}^{1 / 3} s^{*}\right)+B_{0, \mathrm{t}} \operatorname{Bi}\left(-\varphi_{\mathrm{t}}^{1 / 3} s^{*}\right)\right]$.

At the transition point at $r=r_{\mathrm{t}}$, the solution and its first derivative take the values

$$
\left.\begin{array}{l}
\alpha^{(\mathrm{t})}\left(r_{\mathrm{t}} ; \varepsilon\right)=\mu_{0}^{(\mathrm{t})}(\varepsilon) \frac{3^{-2 / 3}}{\Gamma(2 / 3)}\left(A_{0, \mathrm{t}}+\sqrt{3} B_{0, \mathrm{t}}\right), \\
\left(\frac{\mathrm{d} \alpha^{(\mathrm{t})}(r ; \varepsilon)}{\mathrm{d} r}\right)_{r_{\mathrm{t}}} \\
=\frac{\mu_{0}^{(\mathrm{t})}(\varepsilon)}{\varepsilon^{2 / 3}} \frac{3^{1 / 6} \Gamma(2 / 3)}{2 \pi} \varphi_{\mathrm{t}}^{1 / 3}\left(A_{0, \mathrm{t}}-\sqrt{3} B_{0, \mathrm{t}}\right) .
\end{array}\right\}
$$

By the same transformations, Eq. (2) takes the form

$$
\begin{aligned}
v_{0}^{(\mathrm{t})}(\varepsilon) & {\left[\frac{1}{\varepsilon^{4 / 3}} \frac{\mathrm{d}^{2} \xi_{0}^{(\mathrm{t})}}{\mathrm{d} s^{* 2}}+\frac{1}{\varepsilon^{2 / 3}} \frac{4}{r_{\mathrm{t}}+\varepsilon^{2 / 3} s^{*}} \frac{\mathrm{d} \xi_{0}^{(\mathrm{t})}}{\mathrm{d} s^{*}}+\ldots\right] } \\
& +v_{1}^{(\mathrm{t})}(\varepsilon)[\ldots]=\mu_{0}^{(\mathrm{t})}(\varepsilon)\left[\frac{1}{\varepsilon^{2 / 3}} \frac{\mathrm{d} \alpha_{0}^{(\mathrm{t})}}{\mathrm{d} s^{*}}+\ldots\right]+\ldots
\end{aligned}
$$


If one sets

$v_{0}^{(\mathrm{t})}(\varepsilon)=\mu_{0}^{(\mathrm{t})}(\varepsilon)$,

the dominant equation is

$\frac{\mathrm{d}^{2} \xi_{0}^{(\mathrm{t})}}{\mathrm{d} s^{* 2}}=0$

Its solution can be written as

$\xi_{0}^{(\mathrm{t})}\left(s^{*}\right)=C_{0, \mathrm{t}} s^{*}+D_{0, \mathrm{t}}$,

where $C_{0, \mathrm{t}}$ and $D_{0 \mathrm{t}}$ are undetermined constants.

In order to match the first-order asymptotic solutions for $\alpha^{(\mathrm{t})}(r ; \varepsilon)$ and $\alpha^{(\mathrm{o})}(r ; \varepsilon)$, we consider the first-order solution for $\alpha^{(\mathrm{t})}(r ; \varepsilon)$ for large values of $s$ and the first-order solution for $\alpha^{(o)}(r ; \varepsilon)$ for $s \rightarrow 0$. One has that

$$
\begin{aligned}
& \lim _{s \rightarrow \infty} \alpha^{(\mathrm{t})}(r ; \varepsilon)=\frac{\mu_{0}^{(\mathrm{t})}(\varepsilon) \varepsilon^{1 / 6}}{\sqrt{\pi} \varphi_{\mathrm{t}}^{1 / 12} s^{1 / 4}} \\
& \times\left[A_{0, \mathrm{t}} \sin \left(\frac{1}{\varepsilon} \frac{2}{3} \sqrt{\varphi_{\mathrm{t}}} s^{3 / 2}+\frac{\pi}{4}\right)\right. \\
&\left.+B_{0, \mathrm{t}} \cos \left(\frac{1}{\varepsilon} \frac{2}{3} \sqrt{\varphi_{\mathrm{t}}} s^{3 / 2}+\frac{\pi}{4}\right)\right]
\end{aligned}
$$

and, by the use of Taylor Series (15) about $s=0$ and the Taylor series

$\rho(r)=\rho_{\mathrm{t}}+O(s)$

that

$$
\begin{aligned}
& \lim _{s \rightarrow 0} \alpha^{(\mathrm{o})}(r ; \varepsilon)=\frac{1}{\varphi_{\mathrm{t}}^{1 / 4} r_{\mathrm{t}} \sqrt{\rho_{\mathrm{t}}} c_{\mathrm{t}}^{2} s^{1 / 4}} \\
& \quad \times\left[A_{0}^{*} \cos \left(\frac{1}{\varepsilon} \frac{2}{3} \sqrt{\varphi_{\mathrm{t}}} s^{3 / 2}\right)+B_{0}^{*} \sin \left(\frac{1}{\varepsilon} \frac{2}{3} \sqrt{\varphi_{\mathrm{t}}} s^{3 / 2}\right)\right] .
\end{aligned}
$$

By imposing that

$\lim _{s \rightarrow \infty} \alpha^{(\mathrm{t})}(r ; \varepsilon)=\lim _{s \rightarrow 0} \alpha^{(\mathrm{o})}(r ; \varepsilon)$,

one derives the matching conditions

$\mu_{0}^{(\mathrm{t})}(\varepsilon)=\varepsilon^{-1 / 6}$

and

$\left.\begin{array}{l}A_{0}^{*}=\frac{1}{\sqrt{2 \pi}} \varphi_{\mathrm{t}}^{1 / 6} r_{\mathrm{t}} \sqrt{\rho_{\mathrm{t}}} c_{\mathrm{t}}^{2}\left(A_{0, \mathrm{t}}+B_{0, \mathrm{t}}\right), \\ B_{0}^{*}=\frac{1}{\sqrt{2 \pi}} \varphi_{\mathrm{t}}^{1 / 6} r_{\mathrm{t}} \sqrt{\rho_{\mathrm{t}}} c_{\mathrm{t}}^{2}\left(A_{0, \mathrm{t}}-B_{0, \mathrm{t}}\right) .\end{array}\right\}$

One constructs a first-order asymptotic solution for the function $\alpha(r)$ that is uniformly valid from the transition point at $r=r_{\mathrm{t}}$ to a distance sufficiently large from the boundary point at $r=R$, by adding the first-order asymptotic solutions for $\alpha^{(\mathrm{t})}(r ; \varepsilon)$ and $\alpha^{(\mathrm{o})}(r ; \varepsilon)$ and subtracting their common part.
The uniformly valid first-order asymptotic solution can then be expressed as

$$
\begin{aligned}
\alpha^{(\mathrm{t}, \mathrm{u})}(r ; \varepsilon)= & \varepsilon^{-1 / 6}\left[A_{0, \mathrm{t}} \operatorname{Ai}\left(-\varphi_{\mathrm{t}}^{1 / 3} s^{*}\right)+B_{0, \mathrm{t}} \operatorname{Bi}\left(-\varphi_{\mathrm{t}}^{1 / 3} s^{*}\right)\right] \\
& +\frac{1}{\sqrt{2 \pi}} \varphi_{\mathrm{t}}^{1 / 6} r_{\mathrm{t}} \sqrt{\rho_{\mathrm{t}}} c_{\mathrm{t}}^{2} h(r) \\
& \times\left[\left(A_{0, \mathrm{t}}+B_{0, \mathrm{t}}\right) \cos \tau+\left(A_{0, \mathrm{t}}-B_{0, \mathrm{t}}\right) \sin \tau\right] \\
& -\frac{1}{\sqrt{\pi} \varphi_{\mathrm{t}}^{1 / 12} s^{1 / 4}}\left[A_{0, \mathrm{t}} \sin \left(\frac{1}{\varepsilon} \frac{2}{3} \sqrt{\varphi_{\mathrm{t}}} s^{3 / 2}+\frac{\pi}{4}\right)\right. \\
& \left.+B_{0, \mathrm{t}} \cos \left(\frac{1}{\varepsilon} \frac{2}{3} \sqrt{\varphi_{\mathrm{t}}} s^{3 / 2}+\frac{\pi}{4}\right)\right] .
\end{aligned}
$$

With regard to the radial component of the Lagrangian displacement, one observes that Solution (12) for $\xi^{(o)}(r ; \varepsilon)$ and Solution (28) for $\xi_{0}^{(\mathrm{t})}\left(s^{*}\right)$ cannot be matched. We therefore set

$C_{0}^{*}=0, \quad D_{0}^{*}=0, \quad C_{0, \mathrm{t}}=0, \quad D_{0, \mathrm{t}}=0$.

\section{The asymptotic solution in the region near the turning point located on the outside of the acoustic cavity}

In the small region near the turning point that is located on the outside of the acoustic cavity, we introduce the coordinates

$s_{i}=-s=r_{\mathrm{t}}-r, \quad s_{i}^{*}=\frac{s_{i}}{\delta_{i}(\varepsilon)}$,

and asymptotic expansions of the form

$\left.\begin{array}{rl}\alpha^{\left(\mathrm{t}_{i}\right)}(r ; \varepsilon)= & \mu_{0}^{\left(\mathrm{t}_{i}\right)}(\varepsilon) \alpha_{0}^{\left(\mathrm{t}_{i}\right)}\left(s_{i}^{*}\right)+\mu_{1}^{\left(\mathrm{t}_{i}\right)}(\varepsilon) \alpha_{1}^{\left(\mathrm{t}_{i}\right)}\left(s_{i}^{*}\right) \\ & +\ldots, \\ \xi^{\left(\mathrm{t}_{i}\right)}(r ; \varepsilon)= & v_{0}^{\left(\mathrm{t}_{i}\right)}(\varepsilon) \xi_{0}^{\left(\mathrm{t}_{i}\right)}\left(s_{i}^{*}\right)+v_{1}^{\left(\mathrm{t}_{i}\right)}(\varepsilon) \xi_{1}^{\left(\mathrm{t}_{i}\right)}\left(s_{i}^{*}\right) \\ & +\ldots\end{array}\right\}$

After substitution into Eq. (1), one is led to set

$\delta_{i}(\varepsilon)=\varepsilon^{2 / 3}$

and derives the dominant equation

$\frac{\mathrm{d}^{2} \alpha_{0}^{\left(\mathrm{t}_{i}\right)}}{\mathrm{d} s_{i}^{* 2}}-\varphi_{\mathrm{t}} s_{i}^{*} \alpha_{0}^{\left(\mathrm{t}_{i}\right)}=0$.

A general solution of it is given by

$\alpha_{0}^{\left(\mathrm{t}_{i}\right)}\left(s_{i}^{*}\right)=A_{0, \mathrm{t}_{i}} \operatorname{Ai}\left(\varphi_{\mathrm{t}}^{1 / 3} s_{i}^{*}\right)+B_{0, \mathrm{t}_{i}} \operatorname{Bi}\left(\varphi_{\mathrm{t}}^{1 / 3} s_{i}^{*}\right)$,

where $A_{0, t_{i}}$ and $B_{0, t_{i}}$ are undetermined constants. The first-order asymptotic solution for $\alpha^{\left(\mathrm{t}_{i}\right)}(r ; \varepsilon)$ then takes the form

$\alpha^{\left(\mathrm{t}_{i}\right)}(r ; \varepsilon)=\mu_{0}^{\left(\mathrm{t}_{i}\right)}(\varepsilon)\left[A_{0, \mathrm{t}_{i}} \operatorname{Ai}\left(\varphi_{\mathrm{t}}^{1 / 3} s_{i}^{*}\right)+B_{0, \mathrm{t}_{i}} \operatorname{Bi}\left(\varphi_{\mathrm{t}}^{1 / 3} s_{i}^{*}\right)\right]$.

At the turning point at $r=r_{\mathrm{t}}$, the solution and its first derivative take the values

$$
\left.\begin{array}{l}
\alpha^{\left(\mathrm{t}_{i}\right)}\left(r_{\mathrm{t}} ; \varepsilon\right)=\mu_{0}^{\left(\mathrm{t}_{i}\right)}(\varepsilon) \frac{3^{-2 / 3}}{\Gamma(2 / 3)}\left(A_{0, \mathrm{t}_{i}}+\sqrt{3} B_{0, \mathrm{t}_{i}}\right), \\
\left(\frac{\mathrm{d} \alpha^{\left(\mathrm{t}_{i}\right)}(r ; \varepsilon)}{\mathrm{d} r}\right)_{r_{\mathrm{t}}} \\
=\frac{\mu_{0}^{\left(\mathrm{t}_{\mathrm{i}}\right)}(\varepsilon)}{\varepsilon^{2 / 3}} \frac{3^{1 / 6} \Gamma(2 / 3)}{2 \pi} \varphi_{\mathrm{t}}^{1 / 3}\left(A_{0, \mathrm{t}_{i}}-\sqrt{3} B_{0, \mathrm{t}_{i}}\right) .
\end{array}\right\}
$$


On the grounds of the continuity of the first asymptotic solutions $\alpha^{(\mathrm{t})}(r ; \varepsilon)$ and $\alpha^{\left(\mathrm{t}_{i}\right)}(r ; \varepsilon)$ and of their first derivatives at the turning point at $r=r_{\mathrm{t}}$, it follows that

$\mu_{0}^{\left(\mathrm{t}_{i}\right)}(\varepsilon)=\varepsilon^{-1 / 6}, \quad A_{0, t_{i}}=A_{0, \mathrm{t}}, \quad B_{0, \mathrm{t}_{i}}=B_{0, \mathrm{t}}$.

Hence, the first-order asymptotic solution for $\alpha^{\left(\mathrm{t}_{i}\right)}(r ; \varepsilon)$ can be written as

$\alpha^{\left(\mathrm{t}_{i}\right)}(r ; \varepsilon)=\varepsilon^{-1 / 6}\left[A_{0, \mathrm{t}} \operatorname{Ai}\left(\varphi_{\mathrm{t}}^{1 / 3} s_{i}^{*}\right)+B_{0, \mathrm{t}} \operatorname{Bi}\left(\varphi_{\mathrm{t}}^{1 / 3} s_{i}^{*}\right)\right]$.

\section{The asymptotic solution in the region outside the acoustic cavity that is away from the turning point}

In the region outside the acoustic cavity that is away from the turning point at $r=r_{\mathrm{t}}$, we again use a two-variable expansion procedure. We still consider the radial coordinate $r$ as the slow variable but now adopt

$\tau_{i}(r)=\frac{1}{\varepsilon} \int_{r_{\mathrm{t}}}^{r} \sqrt{-\varphi\left(r^{\prime}\right)} \mathrm{d} r^{\prime}$

as the fast variable. It may be noted that $\tau_{i}(r) \leq 0$ and $\mathrm{d} \tau_{i} / \mathrm{d} r \geq 0$. After transformation of Eqs. (1) and (2) and substitution of asymptotic expansions of the form

$$
\left.\begin{array}{l}
\alpha^{(i)}(r ; \varepsilon)=\mu_{0}^{(i)}(\varepsilon) \\
\quad \times\left[\alpha_{0}^{(i)}\left(\tau_{i}, r\right)+\varepsilon \alpha_{1}^{(i)}\left(\tau_{i}, r\right)+O\left(\varepsilon^{2}\right)\right], \\
\xi^{(i)}(r ; \varepsilon)=\mu_{0}^{(i)}(\varepsilon) \\
\quad \times\left[\xi_{0}^{(i)}\left(\tau_{i}, r\right)+\varepsilon \xi_{1}^{(i)}\left(\tau_{i}, r\right)+O\left(\varepsilon^{2}\right)\right],
\end{array}\right\}
$$

one has, at the lowest orders in $\varepsilon$,

$\frac{\partial^{2} \alpha_{0}^{(i)}}{\partial \tau_{i}^{2}}-\alpha_{0}^{(i)}=0$

$\frac{\partial^{2} \xi_{0}^{(i)}}{\partial \tau_{i}^{2}}=0$

$$
\begin{aligned}
\frac{\partial^{2} \alpha_{1}^{(i)}}{\partial \tau_{i}^{2}}- & \alpha_{1}^{(i)}=-\frac{1}{\sqrt{-\varphi}} \\
\times & {\left[2 \frac{\partial^{2} \alpha_{0}^{(i)}}{\partial r \partial \tau_{i}}+\left(K_{2}+\frac{1}{2} \frac{1}{\varphi} \frac{\mathrm{d} \varphi}{\mathrm{d} r}\right) \frac{\partial \alpha_{0}^{(i)}}{\partial \tau_{i}}+K_{4} \frac{\partial \xi_{0}^{(i)}}{\partial r}\right] . }
\end{aligned}
$$

A general solution of Eq. (48) is given by

$\alpha_{0}^{(i)}\left(\tau_{i}, r\right)=A_{0, i}(r) \exp \left(\tau_{i}\right)+B_{0, i}(r) \exp \left(-\tau_{i}\right)$.

The admissible solution of Eq. (49) is

$\xi_{0}^{(i)}(r)=D_{0, i}(r)$.

In this solution, we have dropped a term $C_{0, i}(r) \tau_{i}$ for the reason that the term is inconsistent, since it can be relabeled as $C_{0, i}^{\prime}(r) / \varepsilon$, and its order in $\varepsilon$ is changed.

By removal of the resonant terms in the right-hand member of the inhomogeneous Eq. (50), one obtains

$A_{0, i}(r)=A_{0, \mathrm{c}} h_{i}(r), \quad B_{0, i}(r)=B_{0, \mathrm{c}} h_{i}(r)$, where

$h_{i}(r)=\left\{[-\varphi(r)]^{1 / 4} r \sqrt{\rho(r)} c^{2}(r)\right\}^{-1}$,

and $A_{0, \mathrm{c}}$ and $B_{0, \mathrm{c}}$ are undetermined constants.

The first-order asymptotic solution for $\alpha^{(i)}(r ; \varepsilon)$ then takes the form

$\alpha^{(i)}(r ; \varepsilon)=\mu_{0}^{(i)}(\varepsilon) h_{i}(r)\left[A_{0, \mathrm{c}} \exp \left(\tau_{i}\right)+B_{0, \mathrm{c}} \exp \left(-\tau_{i}\right)\right]$.

It can be verified that $\tau_{i}(r) \rightarrow-\infty$ as $r \rightarrow 0$. To this end, we define the function $F\left(r^{\prime}\right)$ as

$F\left(r^{\prime}\right)=\int \sqrt{-\varphi\left(r^{\prime}\right)} \mathrm{d} r^{\prime}+C$,

where $C$ is an undetermined constant. According to Definition (46), the fast variable $\tau_{i}(r)$ can then be expressed as

$\tau_{i}(r)=\frac{1}{\varepsilon}\left[F(r)-F\left(r_{t}\right)\right]$.

For small values of $r$, one has

$F(r) \simeq \frac{\ell+1 / 2}{|\sigma|} \ln r$,

so that

$\tau_{i}(r) \simeq\left(\ell+\frac{1}{2}\right) \ln r-\frac{1}{\varepsilon} F\left(r_{\mathrm{t}}\right)$.

Hence, $\tau_{i}(r) \rightarrow-\infty$ as $r \rightarrow 0$.

Furthermore, one observes that, in asymptotic Solution (55),

$\exp \left(\tau_{i}\right) \simeq \exp \left[\left(\ell+\frac{1}{2}\right) \ln r\right]=r^{\ell+1 / 2}$.

Since the function $[-\varphi(r)]^{1 / 4}$ behaves as $r^{-1 / 2}$, the function $h_{i}(r)$ behaves also as $r^{-1 / 2}$. Hence, the particular solution $h_{i}(r) \exp \left(\tau_{i}\right)$ tends to zero as $r^{\ell}$, as is required by the study of the behavior of the eigenfunctions near the singular point at $r=0$. It may be noted that this behavior results from the replacement of the factor $\ell(\ell+1)$ by the factor $(\ell+1 / 2)^{2}$ in Definition (4) of the function $\varphi(r)$.

Similarly, one verifies that the particular solution $h_{i}(r) \exp \left(-\tau_{i}\right)$ behaves as $r^{-(\ell+1)}$ as $r \rightarrow 0$. Since this particular solution is not admissible, we set

$B_{0, \mathrm{c}}=0$.

For the matching of the first-order asymptotic solutions for $\alpha^{\left(\mathrm{t}_{i}\right)}(r ; \varepsilon)$ and $\alpha^{(i)}(r ; \varepsilon)$, we consider the first-order solution for $\alpha^{(i)}(r ; \varepsilon)$ for large values of $s_{i}$ and the first-order solution for $\alpha^{(i)}(r ; \varepsilon)$ for $s_{i} \rightarrow 0$. By passing on to the first asymptotic approximations of the Airy functions for large arguments, one has

$\lim _{s_{i} \rightarrow \infty} \alpha^{\left(\mathrm{t}_{i}\right)}(r ; \varepsilon)=\frac{1}{2 \sqrt{\pi} \varphi_{\mathrm{t}}^{1 / 12} s_{i}^{1 / 4}}$

$\times\left[A_{0, \mathrm{t}} \exp \left(-\frac{1}{\varepsilon} \frac{2}{3} \sqrt{\varphi_{\mathrm{t}}} s_{i}^{3 / 2}\right)+2 B_{0, \mathrm{t}} \exp \left(\frac{1}{\varepsilon} \frac{2}{3} \sqrt{\varphi_{\mathrm{t}}} s_{i}^{3 / 2}\right)\right]$.

On the other hand, from Definition (46), it follows that

$\tau_{i}(r)=-\frac{1}{\varepsilon} \int_{0}^{s_{i}} \sqrt{-\varphi\left(r^{\prime}\right)} \mathrm{d} s_{i}^{\prime}$, 
so that, for small values of $s_{i}$,

$\tau_{i}(r)=-\frac{1}{\varepsilon} \frac{2}{3} \sqrt{\varphi_{\mathrm{t}}} s_{i}^{3 / 2}$

and

$\lim _{s_{i} \rightarrow 0} \alpha^{(i)}(r ; \varepsilon)=\frac{\mu_{0}^{(i)}(\varepsilon)}{\varphi_{\mathrm{t}}^{1 / 4} r_{\mathrm{t}} \sqrt{\rho_{\mathrm{t}}} c_{\mathrm{t}}^{2} s_{i}^{1 / 4}} A_{0, \mathrm{c}} \exp \left(-\frac{1}{\varepsilon} \frac{2}{3} \sqrt{\varphi_{\mathrm{t}}} s_{i}^{3 / 2}\right)$

By imposing that

$\lim _{s_{i} \rightarrow \infty} \alpha^{\left(\mathrm{t}_{i}\right)}(r ; \varepsilon)=\lim _{s_{i} \rightarrow 0} \alpha^{(i)}(r ; \varepsilon)$,

one derives the matching conditions

$\mu_{0}^{(i)}(\varepsilon)=\varepsilon^{0}, \quad A_{0, \mathrm{t}}=\frac{2 \sqrt{\pi}}{\varphi_{\mathrm{t}}^{1 / 6} r_{\mathrm{t}} \sqrt{\rho_{\mathrm{t}}} c_{\mathrm{t}}^{2}} A_{0, \mathrm{c}}, \quad B_{0, \mathrm{t}}=0$.

From Relations (34), it then follows that

$A_{0}^{*}=\sqrt{2} A_{0, \mathrm{c}}, \quad B_{0}^{*}=\sqrt{2} A_{0, \mathrm{c}}$,

so that the constants $A_{0}^{*}$ and $B_{0}^{*}$ in the first-order asymptotic solution for $\alpha^{(0)}(r ; \varepsilon)$ are equal to each other.

The first-order asymptotic solution that is uniformly valid from the central point at $r=0$ to the turning point at $r=r_{\mathrm{t}}$ can be expressed as

$$
\begin{gathered}
\alpha^{\left(\mathrm{t}_{i}, \mathrm{u}\right)}(r ; \varepsilon)=A_{0, \mathrm{t}}\left[\varepsilon^{-1 / 6} \operatorname{Ai}\left(\varphi_{\mathrm{t}}^{1 / 3} s_{i}^{*}\right)\right. \\
+\frac{1}{2 \sqrt{\pi}} \varphi_{\mathrm{t}}^{1 / 6} r_{\mathrm{t}} \sqrt{\rho_{\mathrm{t}}} c_{\mathrm{t}}^{2} h_{i}(r) \exp \left(\tau_{i}\right) \\
\left.-\frac{1}{2 \sqrt{\pi} \varphi_{\mathrm{t}}^{1 / 12} s_{i}^{1 / 4}} \exp \left(-\frac{1}{\varepsilon} \frac{2}{3} \sqrt{\varphi_{\mathrm{t}}} s_{i}^{3 / 2}\right)\right] .
\end{gathered}
$$

\section{The asymptotic solution near $r=R$ and the eigenfrequency equation}

We treat the small region near $r=R$ as a boundary layer. For the construction of the boundary-layer solutions, we pass on from the functions $\alpha(r)$ and $\xi(r)$ to functions $v(r)$ and $w(r)$ by means of the transformation

$$
\left.\begin{array}{l}
\alpha(r)=h(r) v(r) \\
\xi(r)=h(r) \varphi^{-1 / 2}(r) w(r),
\end{array}\right\}
$$

which corresponds formally to the transformation given by Eqs. (39) in Smeyers et al. (1996). One readily obtains the resulting equations from Eqs. (40) and (41) in the same paper by replacing the function $c(r)$ by $\varphi^{-1 / 2}(r)$ and the function $K_{3}(r)$ by $K_{3}^{*}(r)$.

We assume that the mass density, $\rho(r)$, can be expanded in a power series about $r=R$ as

$$
\rho(r)=\rho_{\mathrm{s}} z^{n_{\mathrm{e}}}\left[1+\frac{\rho_{1}}{\rho_{\mathrm{s}}} z+O\left(z^{2}\right)\right],
$$

where $z=R-r$, and $n_{\mathrm{e}}$ is a constant. Furthermore, we assume that, in the surface layers, the mass inside the sphere with radius $r, m(r)$, is almost equal to the star's total mass, $M$, so that the condition of hydrostatic equilibrium leads to a power series for the pressure of the form

$P(r)=P_{\mathrm{s}} z^{n_{\mathrm{e}}+1}\left[1+\frac{P_{1}}{P_{\mathrm{s}}} z+O\left(z^{2}\right)\right]$.

Power series of the following form are then derived for $c(r)$, $\varphi(r)$, and $h(r)$ :

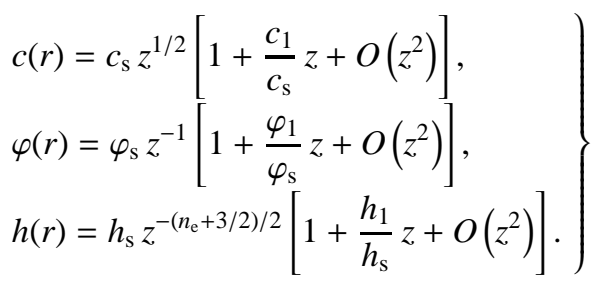

We introduce the boundary-layer coordinate

$\tau_{\mathrm{s}}(z)=\frac{1}{\varepsilon} \int_{0}^{z} \sqrt{\varphi\left(r^{\prime}\right)} \mathrm{d} z^{\prime}$

and boundary-layer expansions of the form

$\left.\begin{array}{l}v^{(\mathrm{s})}(r ; \varepsilon)=\mu_{0}^{(\mathrm{s})}(\varepsilon) v_{0}^{(\mathrm{s})}\left(\tau_{\mathrm{s}}\right)+\mu_{1}^{(\mathrm{s})}(\varepsilon) v_{1}^{(\mathrm{s})}\left(\tau_{\mathrm{s}}\right)+\ldots, \\ w^{(\mathrm{s})}(r ; \varepsilon)=v_{0}^{(\mathrm{s})}(\varepsilon) w_{0}^{(\mathrm{s})}\left(\tau_{\mathrm{s}}\right)+v_{1}^{(\mathrm{s})}(\varepsilon) w_{1}^{(\mathrm{s})}\left(\tau_{\mathrm{s}}\right)+\ldots\end{array}\right\}$

As in Smeyers et al. (1996), the dominant boundary-layer equation for $v_{0}^{(\mathrm{s})}\left(\tau_{\mathrm{s}}\right)$ is

$\frac{\mathrm{d}^{2} v_{0}^{(\mathrm{s})}}{\mathrm{d} \tau_{\mathrm{s}}^{2}}+\left[1-\frac{\left(n_{\mathrm{e}}+1\right)^{2}-1 / 4}{\tau_{\mathrm{s}}^{2}}\right] v_{0}^{(\mathrm{s})}=0$

The equation has the admissible solution

$v_{0}^{(\mathrm{s})}\left(\tau_{\mathrm{s}}\right)=A_{0, \mathrm{~s}} \tau_{\mathrm{s}}^{1 / 2} J_{n_{\mathrm{e}}+1}\left(\tau_{\mathrm{s}}\right)$,

where $J_{n_{\mathrm{e}}+1}\left(\tau_{\mathrm{s}}\right)$ is the Bessel function of the first kind of order $n_{\mathrm{e}}+1$, and $A_{0, \mathrm{~s}}$ an undetermined constant.

For the matching of the first-order asymptotic solution for the function $v^{(\mathrm{s})}(r ; \varepsilon)$ with the first-order asymptotic solution for the function $\alpha^{(\mathrm{o})}(r ; \varepsilon) / h(r)$, one must consider the asymptotic form of the Bessel function $J_{n_{\mathrm{e}}+1}\left(\tau_{\mathrm{s}}\right)$ for large arguments. Taking into account that

$\tau_{\mathrm{s}}(r)=\tau_{R}-\tau(r)$

with

$\tau_{R}=\frac{1}{\varepsilon} \int_{r_{\mathrm{t}}}^{R} \sqrt{\varphi\left(r^{\prime}\right)} \mathrm{d} r^{\prime}$

one performs the matching by setting

$\mu_{0}^{(\mathrm{s})}(\varepsilon)=1$

and

$\left.\begin{array}{l}A_{0}^{*}=A_{0, \mathrm{~s}}\left(\frac{2}{\pi}\right)^{1 / 2} \sin \gamma, \\ A_{0}^{*}=-A_{0, \mathrm{~s}}\left(\frac{2}{\pi}\right)^{1 / 2} \cos \gamma,\end{array}\right\}$

where $\gamma=\tau_{R}-\left(2 n_{\mathrm{e}}+1\right) \pi / 4$. 
The first-order asymptotic solution that is uniformly valid from $r=R$ to a distance sufficiently large from the turning point at $r=r_{\mathrm{t}}$ is then given by

$\alpha^{(\mathrm{s}, \mathrm{u})}(r ; \varepsilon)=A_{0, \mathrm{~s}} h(r) \tau_{\mathrm{s}}^{1 / 2} J_{n_{\mathrm{e}}+1}\left(\tau_{\mathrm{s}}\right)$.

From Equalities (81), it follows that

$A_{0, \mathrm{~s}}(\sin \gamma+\cos \gamma)=0$.

The constant $A_{0, \mathrm{~s}}$ can be different from zero only if

$\sin \gamma+\cos \gamma=0$.

This trigonometric equation leads to the eigenfrequency equation

$|\sigma| \int_{r_{\mathrm{t}}}^{R}\left[\frac{1}{c^{2}(r)}-\frac{(\ell+1 / 2)^{2}}{\sigma^{2} r^{2}}\right]^{1 / 2} \mathrm{~d} r=\left(n+\frac{n_{\mathrm{e}}}{2}\right) \pi$,

where $n$ takes the values $1,2,3, \ldots$ and is the radial order of the $p$-mode considered. In the Cowling classification, the order of a $p$-mode corresponds to the number of nodes of the eigenfunction $\xi(r)$ between $r=0$ and $r=R$ or, equivalently, to the number of nodes plus 1 of the eigenfunction $\alpha(r)$ in the same interval. The degree $\ell$ to which the $p$-mode considered belongs, has an effect on the eigenfrequency through the position of the turning point and the factor $(\ell+1 / 2)^{2}$, which appears in the integrand in the left-hand member.

The integral in eigenfrequency Eq. (85) is equal to the time needed for an acoustic wave to propagate in the radial direction from the turning point to the star's surface with the local velocity of propagation

$V(r)=\frac{c(r)}{\left[1-\frac{(\ell+1 / 2)^{2} c^{2}(r)}{\sigma^{2} r^{2}}\right]^{1 / 2}}$,

which is everywhere larger than the local isentropic sound velocity, except at the star's surface. The velocity of propagation is infinitely large at the turning point and decreases as the radial distance $r$ increases.

For a pair of $p$-modes of a degree $\ell$ and radial order $n$ and of the degree $\ell+2$ and the radial order $n-1$, which have nearly the same eigenfrequency, the approximate relation holds

$\frac{\int_{r_{\mathrm{t}}(\ell)}^{R}\left[\frac{1}{c^{2}(r)}-\frac{(\ell+1 / 2)^{2}}{\sigma^{2} r^{2}}\right]^{1 / 2} \mathrm{~d} r}{\int_{r_{\mathrm{t}}(\ell+2)}^{R}\left[\frac{1}{c^{2}(r)}-\frac{(\ell+5 / 2)^{2}}{\sigma^{2} r^{2}}\right]^{1 / 2} \mathrm{~d} r} \simeq \frac{n+\frac{n_{\mathrm{e}}}{2}}{n-1+\frac{n_{\mathrm{e}}}{2}}$.

The effect of the reduction of the radial order $n$ by one appears to be approximately compensated by a move of the turning point to a somewhat larger radial distance from the centre and by an increase of the local velocity of propagation of the acoustic wave, both phenomena being related to the increase of the degree $\ell$ by two.

Eigenfrequency Eq. (85) can be compared with eigenfrequency Eq. (48) in Roxburgh \& Vorontsov (1996). In the leading-order approximation, the latter eigenfrequency equation reduces to

$|\sigma| \int_{r_{\mathrm{t}}}^{R}\left[\frac{1}{c^{2}(r)}-\frac{(\ell+1 / 2)^{2}}{\sigma^{2} r^{2}}\right]^{1 / 2} \mathrm{~d} r=\left(n+\alpha_{\text {out }}\right) \pi$,

in accordance with the comments given by the authors in their Sect. 4 ( $\alpha$ int is neglected and $B$ is set equal to zero). The value of $\alpha_{\text {out }}$ results from the matching of the solution constructed in the solar outer layers by means of the method of the phase functions, and the solution constructed in the solar interior. From the comparison, it follows that eigenfrequency Eqs. (85) and (88) agree, apart from the difference due to the dissimilarity of the assumptions that are adopted with regard to the outer layers.

\section{Application to the equilibrium sphere with uniform mass density}

In order to examine the improvement in accuracy of the present asymptotic description in comparison with the standard firstorder asymptotic description for low-degree $p$-modes of high radial orders (Tassoul 1990; Smeyers et al. 1996), we have applied eigenfrequency Eq. (85) to a variety of $p$-modes of the equilibrium sphere with uniform mass density. In this equilibrium model, the distributions of the mass density, the pressure, and the isentropic sound velocity are given by

$\left.\begin{array}{l}\rho=\frac{3 M}{4 \pi R^{3}}, \\ P(r)=\frac{3 G M^{2}}{8 \pi R^{4}}\left(1-x^{2}\right), \\ c(r)=\left[\frac{5 G M}{6 R}\left(1-x^{2}\right)\right]^{1 / 2},\end{array}\right\}$

where $x=r / R$. In the expression for the isentropic sound velocity, we have adopted the value $5 / 3$ for the generalized isentropic coefficient $\Gamma_{1} \equiv(\partial \ln P / \partial \ln \rho)_{S}$. Consequently, for a $p$-mode associated with a degree $\ell$ and an eigenfrequency $\sigma$, the distance of the turning point from the centre is given by

$r_{\mathrm{t}}=\left[1+\frac{6 \sigma^{2}}{5(\ell+1 / 2)^{2}}\right]^{-1 / 2}$.

In the equilibrium sphere with uniform mass density, the assumption that, near $r=R$, the mass inside the sphere with radius $r, m(r)$, is nearly equal to the total mass, $M$, does not hold, but the pressure distribution is given in that region by an expression of the form of Taylor Series (72).

For each $p$-mode considered, we have determined the exact eigenfrequency, $\sigma_{\text {exact }}$, by means of the eigenfrequency equation

$\sigma_{\text {exact }}=\left(\frac{G M}{R^{3}}\right)^{1 / 2}\left\{D_{\ell, k}+\left[D_{\ell, k}^{2}+\ell(\ell+1)\right]^{1 / 2}\right\}^{1 / 2}$,

where $k=n-1$, and

$D_{\ell, k}=-2+\Gamma_{1}[k(2 k+5+2 \ell)+3+2 \ell] / 2$. 
(Sauvenier-Goffin 1951; Ledoux \& Walraven 1958, Sect. 76). We have also determined the first asymptotic approximation

$\left|\sigma_{\mathrm{T}}\right|=\frac{\left(2 n+\ell+n_{\mathrm{e}}+\frac{1}{2}\right) \frac{\pi}{2}}{\int_{0}^{R} \frac{\mathrm{d} r}{c(r)}}$

given by the asymptotic theory that applies to low-degree $p$-modes of high radial orders. Furthermore, we have started from this asymptotic approximation in order to solve eigenfrequency Eq. (85) numerically by means of the Newton-Raphson method and to determine the asymptotic approximation $\sigma_{\text {asymp }}$.

The eigenfrequencies $\sigma_{\text {exact }}, \sigma_{\mathrm{T}}$, and $\sigma_{\text {asymp }}$ are presented in Table 1 for the $p$-modes of the radial orders $n=5$, $10,15,20,25,30,35,40$ belonging to the lowest degrees $\ell=1$, $2,3,4$. They are all expressed in the unit $\left(G M / R^{3}\right)^{1 / 2}$. In the same table, the relative radial distance of the turning point from the centre, $r_{\mathrm{t}} / R$, and the relative errors of the asymptotic approximations $\sigma_{\mathrm{T}}$ and $\sigma_{\text {asymp }}$ of the eigenfrequencies are also presented.

For $\ell=1$, the radial orders considered are limited to $n=5,10,15,20,25$, since for $n>25$ the relative error of the asymptotic eigenfrequency $\sigma_{\text {asymp }}$ appears to increase slightly. We ascribe this increase to the fact that, for $p$-modes of radial orders $n$ larger than 25, the turning point is situated so close to the star's centre that it is located in the boundary layer near $r=0$. Under this circumstance, the asymptotic theory for lowdegree $p$-modes of high radial orders should be applied rather than the asymptotic theory developed above.

In the last but three and two columns of Table 1, the exact values and the asymptotic approximations of the small frequency separations $D_{n, \ell}$ are given for the $p$-modes belonging to $\ell=1$ and the $p$-modes belonging to $\ell=2$. The small frequency separations are determined as

$D_{n, \ell}=\frac{1}{2 \ell+3}\left(\sigma_{n, \ell}-\sigma_{n-1, \ell+2}\right)$,

where the eigenfrequencies are still expressed in the unit $\left(G M / R^{3}\right)^{1 / 2}$. In the last column of Table 1 , the relative errors of the asymptotic approximations of the small frequency separations, $\left(D_{n, \ell}\right)_{\mathrm{as}}$, with respect to the exact values, $\left(D_{n, \ell}\right)_{\mathrm{ex}}$, are presented.

Table 1 shows that, for the low-degree $p$-modes considered, both first-order asymptotic approximations of the eigenfrequency are somewhat larger than the exact eigenfrequency, and that the asymptotic eigenfrequencies determined by means of Eq. (85) have values closer to those of the exact eigenfrequencies. Consequently, the relative errors of the asymptotic eigenfrequencies determined by means of Eq. (85) are systematically smaller than those of the eigenfrequencies determined by means of the asymptotic theory for low-degree $p$-modes of high radial orders. For the various low degrees, the relative errors of the eigenfrequencies determined by means of Eq. (85) are already smaller than $0.53 \%$ as $n=10$. For $n=40$, they are of the order of $0.035 \%$.

As the degree $\ell$ increases, the relative errors of the eigenfrequencies resulting from the asymptotic theory for low-degree

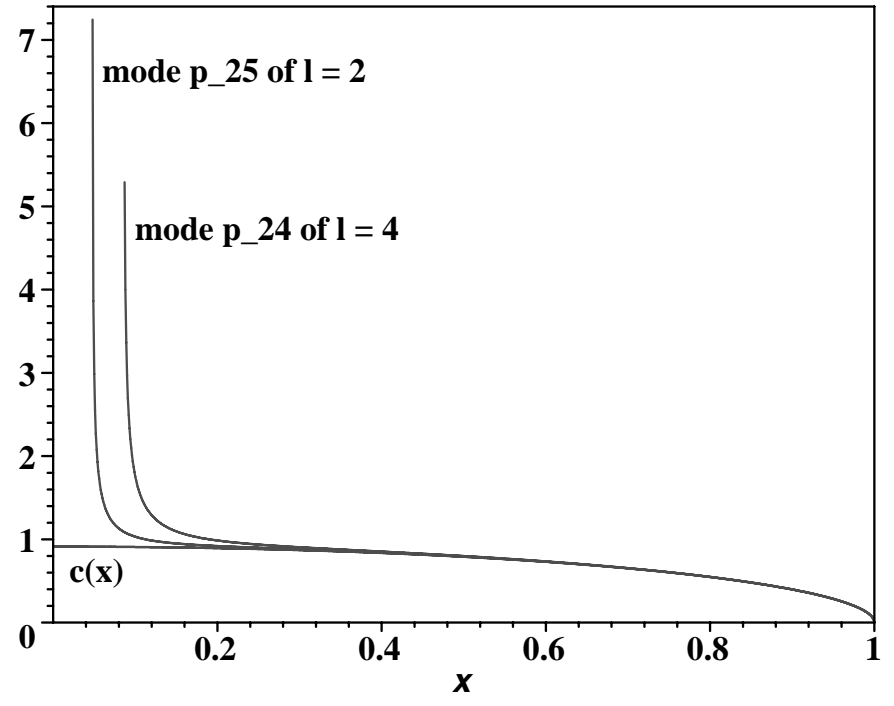

Fig. 1. Variation of the velocity of propagation as defined by Eq. (86) for the mode $p_{25}$ belonging to $\ell=2$ and the mode $p_{24}$ belonging to $\ell=4$, and variation of the isentropic sound velocity, as functions of the relative radial distance $x=r / R$. The velocities are expressed in the unit $(G M / R)^{1 / 2}$

$p$-modes of high radial orders are seen to increase, as can be expected, while the relative errors of the eigenfrequencies resulting from Eq. (85) decrease slightly. The ratios of the relative errors are approximately 1.46 for the modes belonging to $\ell=1$, approximately 2.29 for the modes belonging to $\ell=2$, approximately 3.55 for the modes belonging to $\ell=3$, and approximately 5.22 for the modes belonging to $\ell=4$.

From the last three columns of Table 1, it appears that, for $\ell=1$ and $\ell=2$, the asymptotic approximations of the small frequency separations, $\left(D_{n, \ell}\right)_{\text {as }}$, are somewhat smaller than the exact values, $\left(D_{n, \ell}\right)_{\mathrm{ex}}$, so that the relative errors are negative. Already from the radial order $n=15$ on, the absolute value of the relative error of the asymptotic approximation of the small frequency separation $D_{n, \ell}$ is smaller than $0.1 \%$.

In order to throw light on the origin of the small frequency separations $D_{n, \ell}$, we have represented the variation of the velocity of propagation as given by Eq. (86), for the mode $p_{25}$ of $\ell=2$, with eigenfrequency $\sigma_{\text {asymp }}=47.8714$, and for the mode $p_{24}$ of $\ell=4$, with eigenfrequency $\sigma_{\text {asymp }}=47.7493$. For the first mode, the turning point is located at $r_{\mathrm{t}} / R=0.04762$, for the second mode, at $r_{\mathrm{t}} / R=0.08571$. The variations of the velocities of propagation as well as the variation of the isentropic sound velocity are represented in Fig. 1. For both $p$ modes, the velocity of propagation sharply decreases close to the turning point and tends rapidly towards the isentropic sound velocity, so that the velocities of propagation are nearly identical from $x \simeq 0.28$ to $x=1$. The figure illustrates that the increase in the degree $\ell$ by two mainly induces a move of the turning point to a somewhat larger distance from the centre. From this, we infer that, apart from the difference in the radial orders of the modes, the region situated in the vicinity of the two turning points contributes significantly to the formation of the small frequency separation $D_{25,2}$. This conclusion agrees with an 
Table 1. Exact eigenfrequencies $\sigma_{\text {exact }}$, and first-order asymptotic approximations $\sigma_{\mathrm{T}}$ and $\sigma_{\text {asymp }}$ and their relative errors for low-degree $p$-modes of the equilibrium sphere with uniform mass density. For the degrees $\ell=1$ and $\ell=2$, exact small frequency separations $D_{n, \ell}$, and first-order asymptotic approximations and their relative errors are also given.

\begin{tabular}{|c|c|c|c|c|c|c|c|c|c|c|}
\hline$\ell$ & $n$ & $\sigma_{\text {exact }}$ & $r_{\mathrm{t}} / R$ & $\sigma_{\mathrm{T}}$ & $\begin{array}{c}\text { rel. err. } \\
\%\end{array}$ & $\sigma_{\text {asymp }}$ & $\begin{array}{c}\text { rel. err. } \\
\%\end{array}$ & $\left(D_{n, \ell}\right)_{\mathrm{ex}}$ & $\left(D_{n, \ell}\right)_{\text {as }}$ & $\begin{array}{c}\text { rel. err. } \\
\%\end{array}$ \\
\hline \multirow[t]{5}{*}{1} & 5 & 10.2153 & 0.1329 & 10.4980 & 2.767 & 10.4083 & 1.890 & 0.08220 & 0.08167 & -0.64 \\
\hline & 10 & 19.4766 & 0.07013 & 19.6267 & 0.7707 & 19.5789 & 0.5252 & 0.04288 & 0.04280 & -0.20 \\
\hline & 15 & 28.6531 & 0.04773 & 28.7554 & 0.3570 & 28.7228 & 0.2432 & 0.02911 & 0.02909 & -0.096 \\
\hline & 20 & 37.8065 & 0.03620 & 37.8841 & 0.2053 & 37.8594 & 0.1398 & 0.02206 & 0.02204 & -0.055 \\
\hline & 25 & 46.9503 & 0.02915 & 47.0129 & 0.1332 & 46.9929 & 0.09068 & 0.01776 & 0.01775 & -0.030 \\
\hline \multirow[t]{8}{*}{2} & 5 & 11.0023 & 0.2031 & 11.4109 & 3.714 & 11.1803 & 1.619 & 0.07675 & 0.07636 & -0.51 \\
\hline & 10 & 20.3146 & 0.1116 & 20.5396 & 1.108 & 20.4124 & 0.4817 & 0.04119 & 0.04111 & -0.18 \\
\hline & 15 & 29.5128 & 0.07710 & 29.6683 & 0.5268 & 29.5804 & 0.2290 & 0.02829 & 0.02827 & -0.089 \\
\hline & 20 & 38.6782 & 0.05890 & 38.7970 & 0.3072 & 38.7298 & 0.1335 & 0.02157 & 0.02156 & -0.053 \\
\hline & 25 & 47.8296 & 0.04766 & 47.9257 & 0.2010 & 47.8714 & 0.08733 & 0.01744 & 0.01743 & -0.035 \\
\hline & 30 & 56.9737 & 0.04002 & 57.0544 & 0.1417 & 57.0088 & 0.06157 & 0.01463 & 0.01463 & -0.024 \\
\hline & 35 & 66.1135 & 0.03450 & 66.1831 & 0.1053 & 66.1438 & 0.04573 & 0.01261 & 0.01261 & -0.018 \\
\hline & 40 & 75.2507 & 0.03031 & 75.3119 & 0.08127 & 75.2773 & 0.03530 & 0.01108 & 0.01108 & -0.013 \\
\hline \multirow[t]{8}{*}{3} & 5 & 11.7369 & 0.2627 & 12.3238 & 5.001 & 11.9024 & 1.410 & & & \\
\hline & 10 & 21.1193 & 0.1496 & 21.4525 & 1.577 & 21.2132 & 0.4444 & & & \\
\hline & 15 & 30.3482 & 0.1047 & 30.5812 & 0.7677 & 30.4138 & 0.2162 & & & \\
\hline & 20 & 39.5307 & 0.08056 & 39.7099 & 0.4533 & 39.5811 & 0.1277 & & & \\
\hline & 25 & 48.6930 & 0.06548 & 48.8386 & 0.2991 & 48.7340 & 0.08421 & & & \\
\hline & 30 & 57.8447 & 0.05515 & 57.9673 & 0.2120 & 57.8792 & 0.05970 & & & \\
\hline & 35 & 66.9901 & 0.04764 & 67.0960 & 0.1581 & 67.0199 & 0.04453 & & & \\
\hline & 40 & 76.1315 & 0.04193 & 76.2247 & 0.1225 & 76.1577 & 0.03448 & & & \\
\hline \multirow[t]{8}{*}{4} & 5 & 12.4283 & 0.3138 & 13.2366 & 6.504 & 12.5831 & 1.245 & & & \\
\hline & 10 & 21.8946 & 0.1844 & 22.3653 & 2.150 & 21.9848 & 0.4120 & & & \\
\hline & 15 & 31.1612 & 0.1307 & 31.4940 & 1.068 & 31.2250 & 0.2047 & & & \\
\hline & 20 & 40.3652 & 0.1012 & 40.6228 & 0.6382 & 40.4145 & 0.1223 & & & \\
\hline & 25 & 49.5413 & 0.08264 & 49.7515 & 0.4242 & 49.5816 & 0.08129 & & & \\
\hline & 30 & 58.7027 & 0.06981 & 58.8802 & 0.3023 & 58.7367 & 0.05794 & & & \\
\hline & 35 & 67.8553 & 0.06043 & 68.0089 & 0.2264 & 67.8847 & 0.04338 & & & \\
\hline & 40 & 77.0022 & 0.05327 & 77.1376 & 0.1758 & 77.0281 & 0.03370 & & & \\
\hline
\end{tabular}

earlier conclusion of van Hoolst \& Smeyers (1991) with regard to small frequency separations in a polytropic model.

We have also used Eq. (85) for the determination of asymptotic eigenfrequencies of $p$-modes belonging to intermediate degrees. The results for the $p$-modes of radial orders $n=$ $10,20,30,40$ belonging to the degrees $\ell=10,20,50$ are presented in Table 2 . The eigenfrequencies are again expressed in the unit $\left(G M / R^{3}\right)^{1 / 2}$. In the various cases considered, the relative error of the asymptotic eigenfrequency, $\sigma_{\text {asympt }}$, with respect to the exact eigenfrequency, $\sigma_{\text {exact }}$, is smaller than $0.3 \%$. One observes that, for a given degree $\ell$, the relative error decreases as the radial order $n$ of the $p$-mode increases, and that, for $p$-modes of a given radial order $n$, the relative error decreases as the degree $\ell$ increases.

By way of illustration, we have constructed the first-order asymptotic solution for the eigenfunction $\alpha(r)$ of the $p$-mode of degree $\ell=10$ and radial order $n=10$. The asymptotic solution is displayed in Fig. 2, from $x=0$ to $x=0.9931$, as a function of the relative radial distance $x=r / R$. In the asymptotic approximation, the turning point is situated at $x=0.3443$.

We first constructed the part of the asymptotic solution in the region extending from $x=1$ to $x=0.8521$ by means of 
Table 2. Exact eigenfrequencies $\sigma_{\text {exact }}$, and first-order asymptotic approximations $\sigma_{\text {asymp }}$ and their relative errors, for intermediate-degree $p$-modes in the equilibrium sphere with uniform mass density.

\begin{tabular}{ccllll}
\hline \hline$\ell$ & $n$ & $\sigma_{\text {exact }}$ & $r_{\mathrm{t}} / R$ & $\sigma_{\text {asymp }}$ & $\begin{array}{c}\text { rel. err. } \\
\%\end{array}$ \\
\hline 10 & 10 & 26.0671 & 0.3451 & 26.1406 & 0.2820 \\
& 20 & 45.0487 & 0.2081 & 45.0925 & 0.09717 \\
& 30 & 63.6084 & 0.1490 & 63.6396 & 0.04908 \\
& 40 & 82.0326 & 0.1161 & 82.0569 & 0.02959 \\
\hline \multirow{2}{*}{20} & 10 & 31.8289 & 0.5068 & 31.8852 & 0.1768 \\
& 20 & 51.9245 & 0.3391 & 51.9615 & 0.07127 \\
& 30 & 71.0358 & 0.2548 & 71.0634 & 0.03880 \\
& 40 & 89.7926 & 0.2040 & 89.8146 & 0.02448 \\
\hline \multirow{2}{*}{50} & 10 & 44.8769 & 0.7165 & 44.9073 & 0.06785 \\
& 20 & 68.5313 & 0.5582 & 68.5565 & 0.03680 \\
& 30 & 89.7013 & 0.4571 & 89.7218 & 0.02284 \\
& 40 & 109.831 & 0.3870 & 109.848 & 0.01570 \\
\hline
\end{tabular}

the uniformly valid solution $\alpha^{(\mathrm{s}, \mathrm{u})}(r ; \varepsilon)$ given by Eq. (82). The choice of the point at $x=0.8521$ as a fitting point with the internal solution is motivated by the fact that, at this point, $\tau_{\mathrm{s}}(r)$ is nearly equal to $\tau_{R} / 2$. We set the asymptotic solution equal to 1 at $x=1$ by imposing that

$A_{0, \mathrm{~s}}=\left(\frac{5}{3}\right)^{3 / 2}\left(\frac{3}{2}\right)^{1 / 2} \frac{\Gamma(2)}{\sigma^{3 / 2}}$.

The asymptotic solution is seen to vary largely in the boundary layer near $x=1$.

Next, we constructed the part of the asymptotic solution that starts outwards from the turning point. To this end, we used the uniformly valid solution $\alpha^{(\mathrm{t}, \mathrm{u})}(r ; \varepsilon)$ given by Eq. (35). The factor $\varphi_{\mathrm{t}}$, which is introduced as a coefficient in the second Taylor Expansion (15), turned out to be equal to 8.972. We fixed the constant $A_{0, \mathrm{t}}$ in terms of the constant $A_{0, \mathrm{~s}}$ by eliminating the constant $A_{0}^{*}$ between the first Eq. (34), in which $B_{0, \mathrm{t}}=0$, and the first Eq. (81). It resulted that $A_{0, \mathrm{t}}=-0.04416$. The firstorder asymptotic approximation for the eigenfunction $\alpha(r)$ displays nine nodes between the turning point at $x=x_{\mathrm{t}}$ and the boundary point at $x=1$, as is expected for a $p$-mode of radial order $n=10$ in the usual Cowling classification.

Finally, we constructed the part of the asymptotic solution in the region that extends from the turning point at $x=x_{\mathrm{t}}$ to the central point at $x=0$. To this end, we used the uniformly valid first-order asymptotic solution $\alpha^{\left(\mathrm{t}_{i}, \mathrm{u}\right)}(r ; \varepsilon)$ determined by Eq. (69). This part of the asymptotic solution displays no node and decreases exponentially towards the central point, where it becomes zero.

The first-order asymptotic eigenfunction is so close to the exact analytical eigenfunction, which is determined by a polynomial of degree 9 (Sauvenier-Goffin 1951; Ledoux \& Walraven 1958, Sect. 76), that the two eigenfunctions cannot be distinguished in Fig. 2.

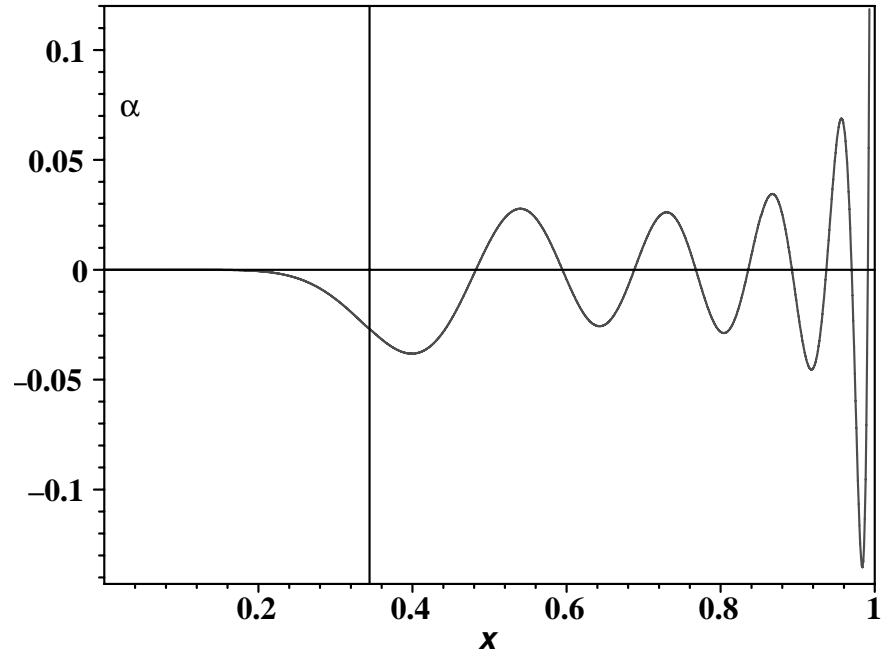

Fig. 2. The first-order asymptotic solution for the eigenfunction $\alpha(r)$ of the $p$-mode of degree $\ell=10$ and radial order $n=10$ in the equilibrium sphere with uniform mass density. The eigenfunction is represented from the relative radial distance $x=0$ to the relative radial distance $x=0.9931$. The vertical line at $x=0.3443$ indicates the position of the turning point in the first-order asymptotic approximation.

\section{Concluding remarks}

We have developed a first-order asymptotic representation of higher-order $p$-modes of stars for which the lower boundary of the resonant acoustic cavity does not lie very close to the star's centre. These $p$-modes are lower-degree $p$-modes associated with lower eigenfrequencies as well as intermediatedegree $p$-modes.

For our purpose, we started from the fourth-order system of differential equations in the radial parts of the divergence and the radial component of the Lagrangian displacement, which was used earlier by Tassoul (1990) and Smeyers et al. (1996) for the construction of an asymptotic representation of lowdegree $p$-modes with high radial orders. Our procedure differs from the procedure adopted in the foregoing investigations mainly by the fact that the lower boundary of the resonant acoustic cavity is no longer considered to be located near the star's centre but to give rise to a turning point in Eq. (1) outside the central boundary layer. The position of the turning point is determined by the zero of the function $\varphi(r)$, in which the parameter $\ell(\ell+1)$ is replaced by the factor $(\ell+1 / 2)^{2}$. By this change, the asymptotic solution for the radial part of the divergence of the Lagrangian displacement, $\alpha(r)$, behaves as $r^{\ell}$ as $r \rightarrow 0$, as is required by the general analysis of the behavior of the eigenfunctions near the singular point at $r=0$. The change is also made in accordance with an earlier remark of Roxburgh \& Vorontsov (1996).

We have constructed the leading-order asymptotic approximation without making any approximation in the fourth-order system of differential equations, in particular with regard to the Eulerian perturbation of the gravitational potential.

The main result is that, in contrast with the first-order asymptotic theory for low-degree $p$-modes of high radial orders, the first-order asymptotic representation developed here 
leads to small frequency separations $D_{n, \ell}$ different from zero. Hence, the inclusion of a turning point in the governing Eqs. (1) and (2) at the lower boundary of the acoustic resonant cavity is of primary importance for the determination of the small frequency separations.

We have tested the validity of the first-order asymptotic representation by applying it to $p$-modes of the equilibrium sphere with uniform mass density, since, for this model, the eigenfrequencies and the eigenfunctions are determined by means of exact analytical solutions.

From Table 1, it appears that the first-order asymptotic eigenfrequencies $\sigma_{\text {asymp }}$ of $p$-modes of lower radial orders belonging to the degrees $\ell=1, \ldots, 4$ are better approximations than are the corresponding eigenfrequencies $\sigma_{\mathrm{T}}$ obtained by the usual asymptotic representation for low-degree $p$-modes of high radial orders. The degree of approximation improves as the value of $\ell$ increases. From the radial order $n=15$ on, the first-order asymptotic approximations of the small frequency separations $D_{n, 1}$ and $D_{n, 2}$ have relative errors that are smaller than $0.1 \%$ in absolute value.

The results presented in Table 2 and the eigenfunction displayed in Fig. 2 confirm the validity of the asymptotic representation for $p$-modes of intermediate degrees. For $\ell=50$, the relative error of the first-order asymptotic eigenfrequency $\sigma_{\text {asymp }}$ is smaller than $0.07 \%$ from the $p$-mode of radial order $n=10 \mathrm{on}$, for which the turning point is situated at $r_{\mathrm{t}} / R=$ 0.7165 .

\section{References}

Kevorkian, J., \& Cole, J. D. 1981, Perturbation Methods in Applied Mathematics (New York: Springer)

Kevorkian, J., \& Cole, J. D. 1996, Multiple Scale and Singular Perturbation Methods (New York: Springer)

Ledoux, P., \& Walraven, Th. 1958, Variable Stars, Handbuch der Physik, 51, 353, ed. S. Flügge (Berlin: Springer)

Kemble, E. C. 1937, The Fundamental Principles of Quantum Mechanics (New York)

Langer, R. E. 1937, Phys. Rev., 51, 669

Lopes, I., \& Turck-Chièze, S. 1994, A\&A, 290, 845

Pekeris, C. L. 1938, ApJ, 88, 189

Roxburgh, I. W., \& Vorontsov, S. V. 1996, MNRAS, 278, 940

Sauvenier-Goffin, E. 1951, Bull. Soc. Roy. Sci. Liège, 20, 20

Smeyers, P., Vansimpsen, T., De Boeck, I., \& van Hoolst, T. 1996, A\&A, 307, 105

Tassoul, M. 1980, ApJS, 43, 469

Tassoul, M. 1990, ApJ, 358, 313

van Hoolst, T., \& Smeyers, P. 1991, A\&A, 248, 647

Vorontsov, S. V. 1991, SvA, 35, 400 